\title{
The Application of Blockchain Technology in Equity Incentive
}

\author{
$\mathrm{Xu} \mathrm{Jia}{ }^{1}$ \\ ${ }^{1}$ Dalian Vocational and Technical College, School of Business Administration, Dalian 116035, Liaoning, China
}

\begin{abstract}
With the advent of the blockchain era, employee incentives need to open up new ideas. The application of blockchain technology can promote the advantages of traditional equity incentive schemes and avoid weaknesses. Although there is still no mature ESOP on-chain solution on the market, and ESOP onchain still needs to consider a series of legal issues. The combination of equity incentives and blockchain technology is feasible. Due to the consensus mechanism of blockchain technology, grant and exercise information is recorded at each node on the chain. The implementation of the plan will be open and transparent throughout the process, avoiding the possibility of dark box operations. This is conducive to enhancing the credibility of the enterprise and enhancing employees' trust in the enterprise, which can really play an incentive role.
\end{abstract}

\section{Introduction}

Equity incentives are Employee Stock Ownership Plans ("ESOP"). The basic feature of ESOP is to enable employees to be the owners of the company at the same time by making employees directly or indirectly hold company shares. ESOP has obvious advantages in improving employee motivation and maintaining employee loyalty. However, the traditional ESOP also shows some shortcomings in practice, such as the procedures for granting, exercising rights and implementing circulation are more complicated.

Many companies have tried equity incentives but most of them have failed. The most important reason for excluding the influence of major external factors such as markets and policies is internal problems of the enterprise. Most companies are always within the framework of the agency system, and have not completely reformed from the perspective of establishing a sharing system, and only use share reform to repair the incentive system based on the pay performance system. This leads to unsatisfactory final results. The result is often that the interests of executives rise, the actual benefits of the company drop significantly, employees resign and cash out, and equity incentives become equity disputes.

\section{Deficiencies in Traditional Equity Incentive Schemes}

Although ESOP has various advantages in improving employee motivation and maintaining employee loyalty, traditional ESOP also reflects deficiencies in practice.

\subsection{The Award Process is More Complicated}

Before granting incentive stock options or options, the company needs to review the employees according to the plan. A complete set of payment processes, including calculation, review and implementation, often rely on a lot of manual operations. If it involves a listed company, it also involves the announcement and registration of the announcement before and after. If the grant process takes a long time, the share price may have experienced several rounds of fluctuation from the decision to the completion of the grant.

\subsection{Exercise Procedures are More Complicated}

If a stock option is granted, there will be a more complicated exercise procedure when the actual exercise takes place. There is generally a process for the delivery of employee exercise notices and company confirmation. If it is a listed company, it involves registration and disclosure. If the original plan involves exercise in stages, the same process must be repeated each time. If ESOP involves a large number of employees, the process will be longer.

\subsection{Complicated to Achieve Circulation}

No matter whether stocks or options are granted to employees, they will face the problem of circulation. For example, when an employee leaves the company, what should he do with the employee stock or employee options, and what should he do if he is not transferred to the designated entity in accordance with the provisions of the award agreement and ESOP? As another example, if employees want to sell stocks after the lock-up period 
expires, can they transfer them out at will? If these stocks or options are not handled properly, it is easy to cause disputes between employees and the company, and even affect the company's shareholding structure, company's stock price or valuation.

In addition, dealing with the differences in performance evaluation, setting the time and conditions for exercise, the advancement and retreat mechanism of employees as shareholders, and the authority of equity incentives are all difficult issues of traditional equity incentives.

\section{The Feasibility of Using Blockchain in Equity Incentives}

The advent of the blockchain era provides a new way for companies to implement employee incentive plans. The blockchain workshop believes that the new idea of using blockchain technology to issue tokens with equity as the underlying right for employee incentives (that is, ESOP on-chain) has feasibility and huge advantages. At present, there are problems in the distribution and management of stock rights of enterprises. Some companies have no way to verify the distribution of options and stock rights. Virtual distribution, oral distribution and other methods cannot protect the legitimate rights and interests of shareholders. Therefore, companies urgently need a way to ensure that employees protect the legitimate rights and interests of shareholders.

Due to the consensus mechanism of blockchain technology, the grant and exercise information is recorded at each node on the chain, and the plan is to implement the entire process of openness and transparency. This not only avoids the possibility of black box operation, but also helps to enhance the credibility of the enterprise, improve the trust of employees in the enterprise, and can really play an incentive role. Blockchain technology can also allow companies to choose the appropriate rights and interests according to their specific circumstances to be on the chain to motivate employees.

Blockchain is essentially a distributed consensus and value incentive technology. On the one hand, it realizes the consensus and trust (rules and transactions) of the entire system through mathematics and algorithms, and on the other hand, it guarantees the value incentive of the ecosystem (wealth record) through tokens. Distributed decentralization, no need for a third-party trust system, and data cannot be tampered with are its main features. Blockchain is divided into public chain, private chain and alliance chain according to the scope of use and the degree of centralization. Due to the non-security of the public chain and the strong centralization of the private chain, the equity trading business based on the blockchain is more carried out on the Consortium Blockchain. The so-called alliance chain refers to the blockchain that is only subject to a part of the designated nodes in the process of establishing node consensus. In principle, only some people can trace all transaction information on the platform. This feature of the alliance chain guarantees weakly centralized collective maintenance, and at the same time does its best to maintain the authority of private information.

Based on the blockchain, we are able to build a safe, credible and self-service equity asset management platform. Besides, we also can complete the online registration of equity information, make a good self-help equity incentive plan design. Moreover, we can do well the employee option online signature grant and equity incentive plan management. Through blockchain technology we can help companies successfully complete equity transactions. By building distributed applications and establishing an end-to-end trust system, it provides investors and investment institutions with an efficient and credible asset circulation environment, allowing equity (bonds) from registration to execution. The data information is continuously recorded on the block and forms a unique digital certificate to ensure the authenticity and integrity of the information; the traceability feature can track the update in real time.

Since equity incentives establish a standard for judging right from wrong, acquiring equity should assume entrepreneurial responsibility. It is not only necessary for the value and significance of the enterprise, including equity incentives to provide financing and financing sources for enterprises, and to build partners in upstream and downstream ecological chains of enterprises through equity incentives. Moreover, it is necessary to prevent and resolve internal disputes among shareholders through equity incentives to create benefits for the company. Blockchain (Bitcoin, Ethereum) and the company's equity incentives have a certain similarity, that is, the product of a network protocol (rule). The network protocol stipulates value (bitcoin, ether) generation rules, transaction rules, data exchange formats, etc. 

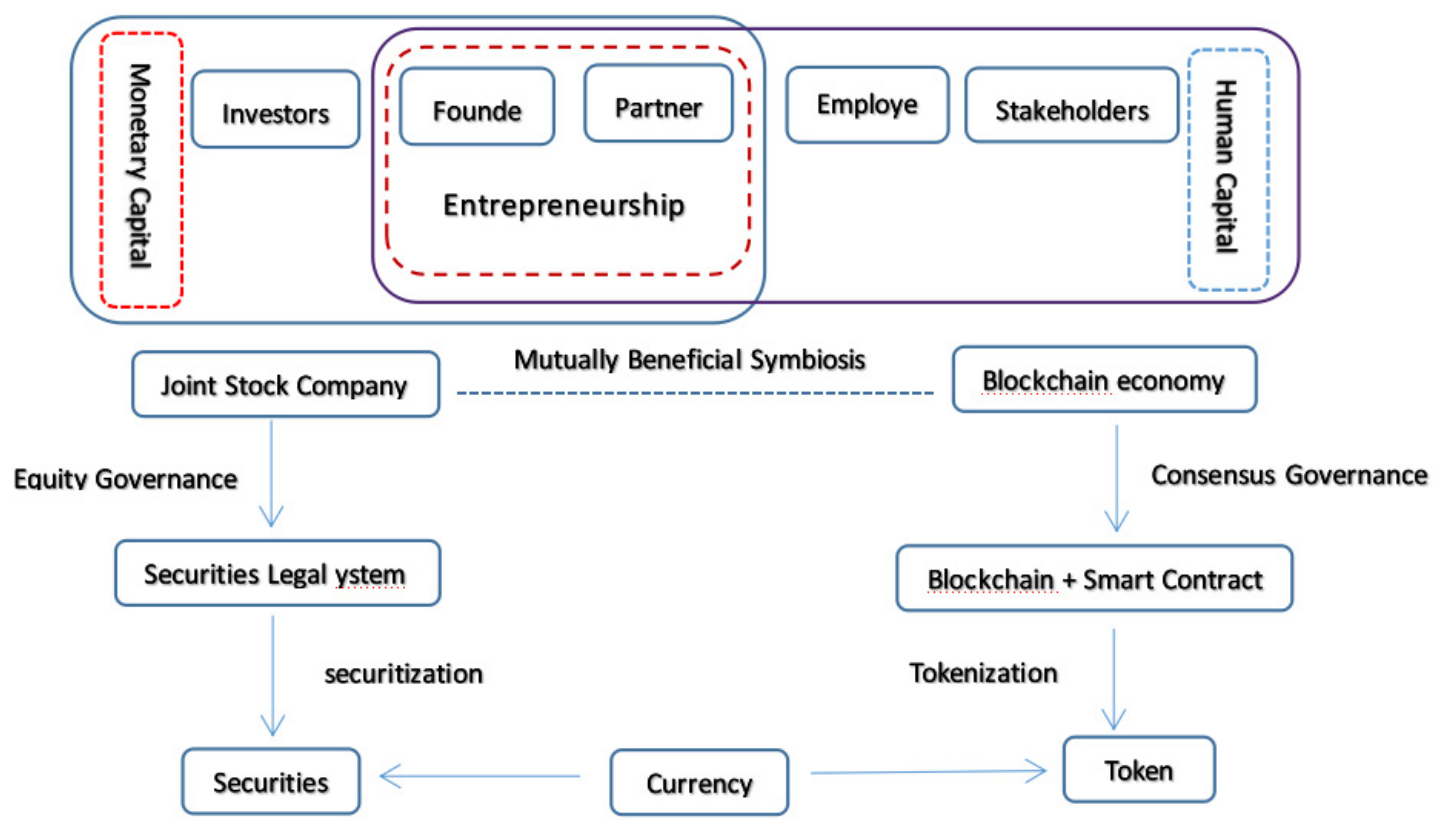

Fig1. Company + Blockchain Economy Framework

As shown in Figure 1, in a blockchain community built on Token, consensus, value, and community are the three core elements. The tokens owned by the participants themselves have room for added value, so that most people in the community hold "In order to increase the value of tokens, members must work hard to build communities and promote community growth."

The future Token economy should rely on blockchain technology to incorporate labor into the wealth accounting system. That is, tokenize human capital, confirm rights, register and flow on the chain, and use the token economy to achieve the optimal allocation of entrepreneurs, technological innovation (talent), labor (employee), and capital (including land). At the same time, China Unicom's currency capital market and human capital market have achieved a new economic equilibrium.

\section{Implementation Design of Equity Incentive Record Management Method Based on Blockchain}

With the development of society, the allocation of options and equity has become a common method for companies to provide benefits for employees, so more and more people begin to pay attention to their options and equity rights.

Current equity allocation and management problems in enterprises. Some companies have no way to verify the distribution of options and equity, such as virtual distribution and oral distribution, which cannot protect the legitimate rights and interests of shareholders.

Therefore, in order to prevent the tampering of the equity information and option information and realize the certification of the employee's equity, it is necessary to design a blockchain-based equity incentive record management method.

The equity incentive record management method based on the blockchain mainly includes: deploying the first smart contract on each node corresponding to the blockchain for equity incentive record management. It will receive the equity allocation request sent from the node, the equity allocation request includes: equity allocation party information, equity recipient information and equity allocation information. Then write the equity distributor information, the equity receiver information and the equity allocation information into the same block. When contract execution is set in the first smart contract, it is verified whether the information of the equity distributor and the information of the equity receiver are valid. When both the information of the equity distributor and the information of the equity receiver are valid, the equity receiver is allocated equity according to the equity allocation information.

Taking a distributed system as a blockchain system as an example, it is formed by multiple nodes (any form of computing devices connected to the network, such as servers and user terminals) and clients. Nodes form a peerto-peer ( $\mathrm{P} 2 \mathrm{P})$ network. The $\mathrm{P} 2 \mathrm{P}$ protocol is an application layer protocol that runs on top of the Transmission Control Protocol (TCP) protocol. In a distributed system, any machine such as a server or terminal can join to become a node. The node includes a hardware layer, an intermediate layer, an operating system layer, and an application layer. The functions of each node in the blockchain system shown in Figure 2 involve the following functions:

Table1. Function Table of Each Node of the Blockchain System

\begin{tabular}{|l|l|}
\hline Function & Fundamental Contents \\
\hline Routing & It has basic functions for supporting communication between nodes. \\
\hline Application & $\begin{array}{l}\text { It is used to deploy in the blockchain, realize specific business according to actual business needs, and record data } \\
\text { related to the realization of functions to form recorded data. Carry a digital signature in the recorded data to indicate }\end{array}$ \\
\hline
\end{tabular}




\begin{tabular}{|l|l|}
\hline Blockchain & $\begin{array}{l}\text { the source of the task data, and send the recorded data to other nodes in the blockchain system. For other nodes to } \\
\text { add the record data to the temporary block when verifying the source and integrity of the record data. }\end{array}$ \\
\hline $\begin{array}{l}\text { It includes a series of blocks that are consecutive to each other in chronological order. Once a new block is added to } \\
\text { the blockchain, it will not be removed. The block records the data submitted by the nodes in the blockchain system. } \\
\text { The blockchain in this application is an equity blockchain. For example, during the equity incentive process, the } \\
\text { equity transaction information of each incentive object is recorded in the blockchain. }\end{array}$ \\
\hline
\end{tabular}

Among them, the services implemented by the application mainly include the following:

(1) Wallet is used to provide the function of trading electronic money. It includes initiating a transaction, that is, sending the transaction record of the current transaction to other nodes in the blockchain system. After the other nodes verify the success, as a response to acknowledge the transaction is valid, and then store the transaction record data in the temporary block of the blockchain. The wallet also supports querying the remaining electronic money in the electronic money address. For example, the information of the incentive objects to be added is sent to the blockchain system. Other nodes in the blockchain system verify the transaction (that is, increase the information of the incentive object in the blockchain). After the other nodes have successfully verified, the transaction can be stored in the blockchain.

(2) Shared ledger is used to provide functions such as storage, query and modification of account data. It sends the recorded data of the operation of the account data to other nodes in the blockchain system. After the other nodes verify the validity, as a response to acknowledge the validity of the account data, the recorded data is stored in a temporary block, and a confirmation can also be sent to the node that initiated the operation. For example, the information of the incentive object and the equity information in the equity incentive are written into the shared ledger after being verified by other nodes, that is, stored in the blockchain.

(3) Smart contract, it is a computerized protocol. It can execute the terms of a contract, implemented by code deployed on the shared ledger to execute when certain conditions are met. The code is used to complete automated transactions according to the actual business requirements, such as querying the logistics status of the goods purchased by the buyer, and after the buyer signs the goods, the buyer's electronic money is transferred to the merchant's address. In addition, smart contracts are not limited to the execution of contracts used for transactions, but can also execute contracts that process received information. For example, when the incentive object meets certain conditions, it will trigger the smart contract to execute the incentive mechanism according to the contract's provisions, such as issuing corresponding benefits or dividends to the incentive object.

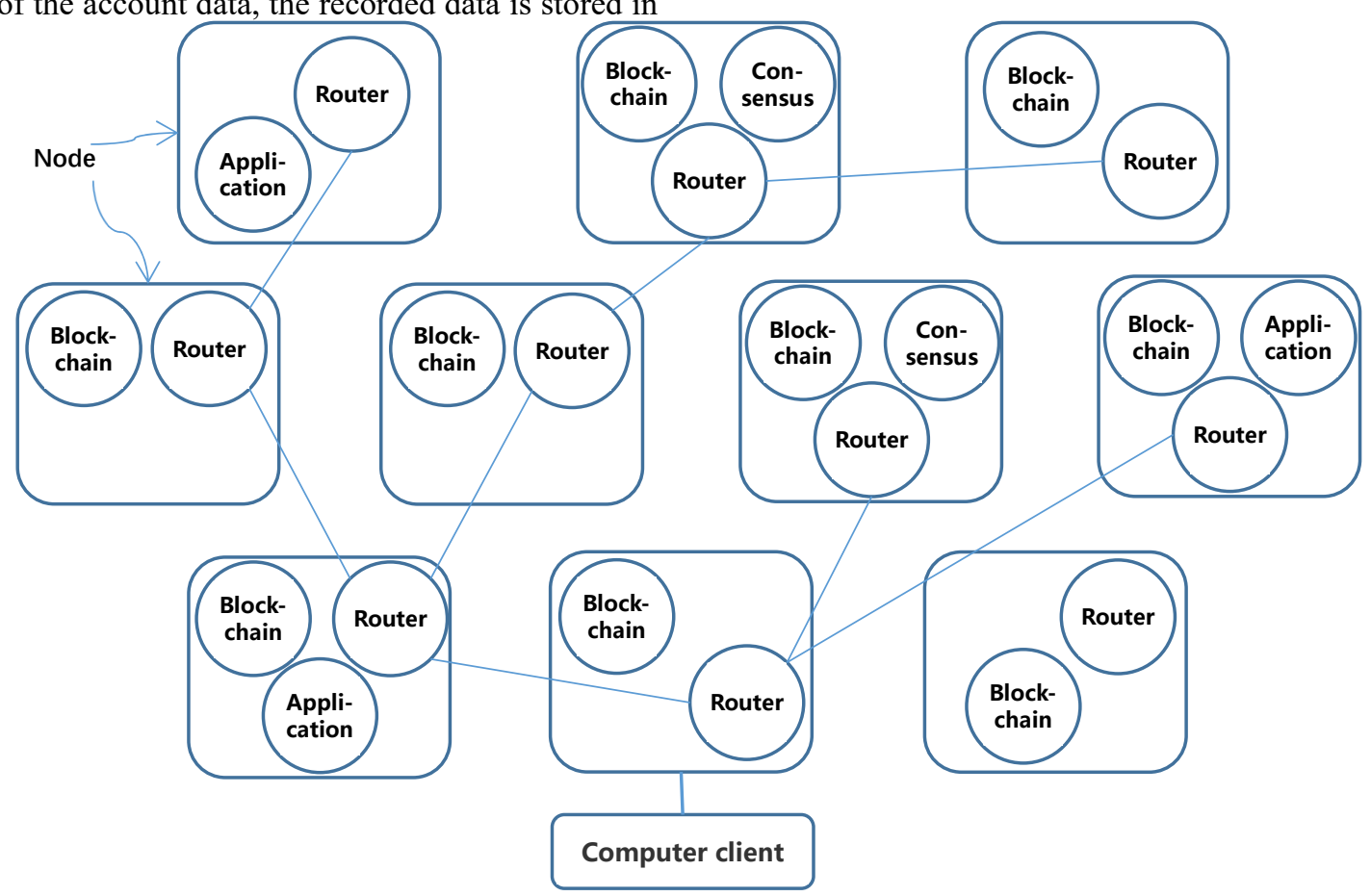

Fig2. Schematic Diagram of Equity Incentive Framework Under Blockchain Technology

Deploy the first smart contract on each node corresponding to the blockchain for equity incentive record management. The triggering conditions of the first smart contract include: one or more of a preset time node, a preset working period, a preset job position, and a preset reward identifier.

(1) First, receive an equity allocation request sent from the node, the equity allocation request includes: equity allocation party information, equity recipient information, and equity allocation information. It will write the information of the equity distributor, the information of the equity receiver and the information of the equity allocation into the same block. When setting the contract execution, the first smart contract verifies whether the information of the equity allocator and the information of the equity receiver are valid. When both the information 
of the equity distributor and the information of the equity receiver are valid, the equity receiver is allocated equity according to the equity allocation information.

Secondly, receiving the equity transfer request sent from the node. The equity transfer request includes equity registration information and equity transfer information, and the equity transfer information is specifically an electronic version of the equity transfer agreement.

Next, the equity registration information and the equity transfer information are written into the same block. When contract execution is set in the first smart contract, after a preset trigger condition is triggered, it is verified whether the equity transfer information is valid according to the equity registration information. When the equity transfer information is valid, the equity transfer clause recorded in the equity transfer agreement corresponding to the equity transfer information is executed.

Subsequently, receiving the equity query message from the user, the equity query message includes: the user's identity information and query condition data, and the query condition data includes: query time range data or keywords. After the identity information is verified, at least one target block is determined according to the user's identity information and the query condition data. The target block is a block containing identity information of the user or equity information of the user corresponding to the query condition data.

Finally, according to the user's identity information and the query condition data, corresponding equity information is obtained and returned from each of the target blocks.

(2) Deploy a second smart contract on each node corresponding to the blockchain. The triggering condition of the second smart contract is that the user's identity information satisfies the preset condition. It is set in the second smart contract that when the user's identity information meets the preset condition, the user's equity transaction limit and holding time limit are limited.

(3) Deploy a third smart contract on each node corresponding to the blockchain. The triggering condition of the third smart contract is to detect that the current moment is the preset equity dividend moment. When setting contract execution in the smart contract, according to the equity registration information in the blockchain, dividends are distributed to the corresponding users, and the dividend information is written into the block.

(4) Deploy a fourth smart contract on each node corresponding to the blockchain. The triggering condition of the fourth smart contract is to detect that the shareholders' working conditions reach the preset conditions. In the fourth smart contract, the shareholder's equity is changed when the contract is executed.

(5) A fifth smart contract is deployed on each node corresponding to the blockchain. Receive the stock transaction request message sent by the transaction requester. The stock transaction request message includes: identity information of the requesting party of the transaction, stock price of the requesting party, number of shares traded by the requesting party, and identity information of the trading object. It is set in the fifth smart contract to verify the identity information of the transaction requester based on the information on the blockchain when the contract is executed. After verification, the stock price of the requesting party and the identity information of the trading object will obtain the stock price of the trading object according to the identity information of the trading object. Then, the trading volume of the trading object is determined according to the number of stock transactions of the requesting party, the stock price of the requesting party and the stock price of the trading object. Finally, the trading volume of the trading object and the stock trading request message are sent to the trading object.

\section{Advantages of Equity Incentives Based on Blockchain Technology}

At present, there is still no mature ESOP on-chain solution on the market, and ESOP on-chain also needs to consider a series of legal issues. For example, how to link ESOP on-chain with the equity registration system, tax planning plan, foreign exchange management system, and securities supervision system. However, the blockchain factory believes that blockchain technology will make the equity incentive plan more effective, and the equity incentive plan is also feasible and has the following advantages.

\subsection{Efficient and Fast Processing}

Compared with traditional schemes, employee incentives share options/complex allocation, exercise and transfer steps. The application of blockchain technology can make employee incentives more efficient and faster. By executing preset smart contracts, employees can automatically obtain tokens for incentives when the conditions are met. Afterwards, whether it is based on the employee's active transfer or the company's transfer requirements for employees after leaving the job, it can ensure that the Token is only transferred in the preset white list. In addition, it does not need to sign complicated transfer agreements, does not require repeated assistance from enterprises, and does not need to change registration through a centralized registration agency, reducing a lot of cumbersome procedures.

\subsection{Increase Employee Trust in the Eompany}

After the employee incentive plan is launched on the chain, due to the consensus mechanism of the blockchain technology, the grant and exercise information is recorded at each node on the chain, and the implementation of the plan will be open and transparent throughout the process. Besides, it is not only traceable and cannot be tampered with, which not only avoids the possibility of black box operations, but also ensures fair, stable and orderly incentives. At the same time, it is conducive to enhancing the credibility of the enterprise, enhancing the trust of employees in the enterprise, and can really play an incentive role. 


\subsection{The Link Between ESOP On-chain and Capital Operation}

If ESOP on-chain and capital operation can be smoothly connected, it can also promote capital operation. If the company plans to implement STO, the ESOP on-chain process will lay a good foundation for STO. On the one hand, the ESOP on-chain process can be regarded as a pathfinding process. Companies need to experience tokenization of incentive shares and smart contractualization of incentive plans. Moreover, a series of operation modes such as the creation and on-chain of Token completed in cooperation with digital solution providers can accumulate experience for STO. On the other hand, if the ESOP has a good effect on the chain, it can also allow future investors to see the company's successful precedent and mature model in the chain reform, and participate more confidently in the company's overall STO.

\subsection{Flexibility}

In the traditional scheme, companies can only use equity or options to motivate employees. However, blockchain technology can allow companies to choose the right equity based on their specific circumstances to be on the chain to motivate employees. In other words, the design of the token can vary from company to company. On the one hand, companies can still design tokens with equity as the underlying fundamental rights and issue them to employees to share capital gains generated by the development of the company. On the other hand, companies can also issue tokens that are not based on equity with other forms of equity, especially those related to products or services that the company itself can provide, to reward employees for their contributions. For example, according to reports, Paypal decided to use blockchain technology to issue crypto tokens to its internal employees for employee incentives. Employees can obtain tokens through the Paypal intranet, participate in related innovation projects and contribute ideas. This kind of token can only be circulated within Paypal, can be traded between employees, and can be exchanged for more than 100 rewards or experiences.

The Token issued under the former circumstances may have the property of securities in some cases because its basic equity is company equity. At this time, it is necessary to pay attention to whether the Token issued to encourage employees is subject to securities supervision in a specific jurisdiction, and if applicable, it should comply with the corresponding regulations. Besides, there is a difference between the tokens issued to motivate employees and the tokens issued by companies for STO for financing purposes. Usually, the scope of circulation is limited to internal and does not involve external investors. In the latter case, the issuance of tokens that are not based on equity is generally not subject to special supervision, and companies can issue them independently.

In addition, for companies that carry out employee stock ownership incentive plans, the original employee stock ownership incentive plans often fall on a single paper document, without a complete digital equity incentive management system. Using blockchain technology, companies can manage various rights such as equity, options, restricted equity, dividend rights, etc. in a decentralized blockchain system. However, employees can control their own share of equity and feel the real incentives. Whether it is a startup or a listed company, a domestic-funded company or a multinational company, employee equity incentive plans are a common means of improving employee motivation and maintaining employee loyalty in corporate management.

\section{Conclusion}

With the advent of the blockchain era, companies need to open up new ideas to motivate employees. The application of blockchain technology can improve the traditional equity incentive schemes and avoid weaknesses. Although, there is still no mature ESOP on-chain solution on the market, and ESOP on-chain needs to consider a series of legal issues. However, the combination of equity incentives and blockchain technology is feasible. The blockchain workshop has been deployed since 2017, and is committed to becoming China's leading comprehensive solution provider for blockchain projects from commercial design to technical implementation. The development of an enterprise is not a struggle alone, or a battlefield in which leaders have sole authority. Enterprises face new environments and new opportunities, and they need to adjust their own development mechanisms in a timely manner to give employees, especially core personnel, sufficient incentives and guidance. In addition, it also can fully integrate human, resources, technology and other advantages, and innovate equity incentive plans. Only in this way can the enterprise make rapid progress in the tide of the times and benefit Changhong. The application of blockchain technology in equity incentives will undoubtedly become the propeller of the enterprise in the process of progress, helping the enterprise to move forward at a rapid speed!

\section{Acknowledgment}

1. Dalian Vocational and Technical College Research and Innovation Fund Project: Research on the Impact of Equity Incentives on Inefficient Investment Behavior of Enterprises（DZ2019CXJJ01)

2. Liaoning Provincial Department of Education's 2019 scientific research funding project: A study on the impact of equity incentive motivation differences on the company's investment and financing behavior (JYT201905)

3. Achievements of Dalian Vocational and Technical College 2019 Scientific research and innovation team

\section{References:}

1. Wang Tao, Zhao Yanyun. Research on Internet Statistics of Product Chain Based on Blockchain[J/OL]. Statistics and Decision, 2020(11): $10-15$ 
2. Chen Hui, Xiang Huahua. Application research of management accounting tools based on blockchain technology [J]. Friends of Accounting, 2020(13):157161.

3. Liu Shuai, Gan Guohua, Liu Mingxi, Fang Yong, Wang Shouyang. A multi-sub-block incentive consensus mechanism based on topology and allocation mechanism design $[\mathrm{J} / \mathrm{OL}]$. Computer Science: 1-21

4. Jin Shuying, Wang Peng, Zhang Zichen. Optimization of accounting information generation path based on blockchain technology $[\mathrm{J}]$. Finance and Accounting Monthly, 2020(11): 76-82.

5. Qu Yang, Qian Beili, Zhang Chengyu, Long Yue, Jia Jie. Design and implementation of an intelligent operation and maintenance system based on blockchain technology $[\mathrm{J}]$. Telecommunications Science, 2020, 36(05): 152- 158. 